\title{
Uso dos condroprotetores na afecção articular degenerativa: revisão
}

\author{
Use of chondroprotectors in degenerative articular disease: review
}

\author{
Alexandre Navarro Alves de Souza ${ }^{[a]}$, Alexandre de Oliveira Saladino ${ }^{[b]}$, Caio Biasi ${ }^{[c]}$, \\ Julia Maria Matera ${ }^{[\mathrm{d}]}$
}

[a] Médico veterinário, Mestre, Universidade de São Paulo (USP), São Paulo, SP - Brasil. e-mail: alexandrenavarro@usp.br

[b] Médico veterinário, Mestre, Universidade de São Paulo (USP), São Paulo, SP - Brasil. e-mail: saladinovet@gmail.com

[c] Médico veterinário, Mestre, Universidade de São Paulo (USP), São Paulo, SP - Brasil. e-mail: caiobiasi@usp.br

[d] Médica veterinária, professora titular do Departamento de Cirurgia da Universidade de São Paulo (USP), São Paulo, SP - Brasil. e-mail: materajm@usp.br

\section{Resumo}

Esta revisão teve como objetivo elucidar e verificar o uso de condroprotetores comumente utilizados no tratamento conservativo para afecções articulares degenerativas em cães. Os critérios de inclusão se concentraram em estudos que avaliaram os condroprotetores mais empregados, como sulfato de condroitina e glucosamina, publicados em revistas científicas. Constatou-se a comprovação experimental da ação dos condroprotetores em alguns dos trabalhos prospectivos analisados, bem como uma divergência entre os demais. A segurança dessa medicação foi avaliada em dois estudos, porém nenhuma das demais pesquisas constatou efeitos adversos importantes. Os condroprotetores mostram boa segurança e efeitos benéficos sobre o metabolismo articular. No entanto, a eficácia clínica permanece discutível graças à metodologia diversificada empregada nos trabalhos científicos.

Palavras-chave: Afecção articular degenerativa. Cão. Condroprotetores.

\begin{abstract}
This research was performed to elucidate and evaluate the use of chondroprotectors in the conservative treatment for degenerative joint diseases in dogs. The inclusion criteria was focused on studies that evaluate the chondroprotectors, like as glucosamine and chondroitin sulphate, published in scientific journals. An analysis of prospective randomized double blind studies find the experimental evidence of the action of these two chondroprotectors and a
\end{abstract}

Rev. Acad., Ciênc. Agrár. Ambient., Curitiba, v. 8, n. 3, p. 281-289, jul./set. 2010 
difference between the others. The safety of this medication was evaluated in two studies, but the others studies not found significant adverse effects. The chondroprotectors show good safety and beneficial effects in joint metabolism. However, clinical effectiveness remains questionable due to the methodology of the researches.

Keywords: Degenerative joint disease. Dog. Chondroprotectors.

\section{Introdução}

A afecção articular degenerativa (AAD), ou osteoartrose (OA), é uma doença de múltiplas causas e caracteriza-se por alterações patológicas da articulação sinovial (BUDSBERG; BARTGES, 2006). A AAD afeta muitas espécies animais e é comum em seres humanos (DAVIS, 1998), com ocorrência estimada em 20\% dos cães com mais de 1 ano de idade (JOHNSTON, 1997).

Essa afecção ocorre graças ao resultado de eventos mecânicos e biológicos que desestabilizam o equilíbrio normal entre a degradação e síntese dos condrócitos da cartilagem articular, matriz extracelular e osso subcondral, culminando na degeneração articular (DEAN; AZZO; MARTEL-PELLETIER, 1987). As evidências clínicas da OA são caracterizadas por dor articular, limitação e alteração dos movimentos, redução do apoio, crepitação, efusão ocasional e graus variáveis de inflamação local, sem efeitos sistêmicos (KEUT'TNER; GOLDBERG, 1995).

Os tratamentos de OA visam ao alívio do desconforto e retardo do desenvolvimento das alterações degenerativas. O tratamento pode ser cirúrgico ou conservativo, dependendo do caso. Não há tratamento que possa evitar ou reverter as alterações patológicas. O tratamento conservativo com anti-inflamatórios não esteroidais (AINEs) é eficaz no início para alívio dos sintomas clínicos decorrentes da inflamação inibindo as prostaglandinas (PGs). Entretanto, segue-se o desconforto após suspensão (LIPOWITZ, 1998).

Dentre os tratamentos alternativos, e que podem ser usados em combinação com AINEs, comumente são utilizados os condroprotetores, que retardam a progressão da AAD (LIPPIELLO et al., 2000), modulando o metabolismo da cartilagem articular (JOHNSON et al., 2001).

Muitos estudos avaliaram a eficácia dos condroprotetores sob diferentes aspectos, seja por escores subjetivos da sintomatologia clínica (DOBENECKER; BEETZ; KIENZE, 2002; GINGERICH; STROBEL, 2003; McCARTHY et al., 2007), mensurações de mudanças bioquímicas e biológicas dos componentes articulares (LIPPIELLO et al., 2000; JOHNSON et al., 2001; NEIL; CARON; ORTH, 2005), alterações hematológicas e da bioquímica sanguínea (McNAMARA; BARR; ERB, 1996) ou até por métodos quantitativos, por meio da análise cinética das forças de reação ao solo (MOREAU et al., 2003).

Visando a esclarecer o efeito dos condroprotetores mais utilizados em cães com AAD, realizou-se uma revisão de literatura sobre os conceitos básicos e os resultados obtidos com o uso destes, a partir de trabalhos que avaliaram esses nutracêuticos.

\section{Etiopatogenia}

Diversos componentes são afetados na $\mathrm{AO}$, como a cartilagem articular, cápsula e líquido sinovial, assim como o osso subcondral. De acordo com o estágio de progressão da AO, os condrócitos sofrem modificações, transformando-se em condrócitos hipertróficos ou em células fagocitárias, ou sofrem a morte por apoptose. A partir desses eventos ocorrem então a hipertrofia, a degradação e a mineralização da cartilagem. $\mathrm{Na}$ OA induzida experimentalmente foi observada a hipertrofia da cartilagem de modo precoce, indicando que os condrócitos possuem capacidade de reparação (BRAUNSTEIN et al., 1990; KUSUZAKI et al., 2001).

Durante a fase de reparação, os agrupamentos de condrócitos começam a produzir quantidades grandes de proteoglicanos e colágeno tipo II, bem como moléculas de colágeno tipo I, II, III e X e tenascina (YASUDA; POOLE, 2002). Esta superprodução de componentes da matriz leva à hipertrofia da cartilagem

Rev. Acad., Ciênc. Agrár. Ambient., Curitiba, v. 8, n. 3, p. 281-289, jul./set. 2010 
e, apesar da tentativa de reparação, uma perda líquida de cartilagem é observada posteriormente ao decorrer da progressão da doença, sugerindo que o reparo da cartilagem é uma reação transitória e ineficaz (KIRSCH; SWOBODA; NAH, 2000; AIGNER et al., 2001). Na fase mais agressiva da doença, os condrócitos produzem níveis mais elevados de metaloproteinases e menores níveis de moléculas da matriz da cartilagem. Este desequilíbrio entre o catabolismo e anabolismo é responsável pela degradação progressiva da matriz (BLUTEAU et al., 2001; TETLOW; ADLAM; WOOLLEY, 2001).

A explicação para o fracasso da reparação ainda é incerta, entretanto, foi observado que há o aumento de diversos fatores ligados à degradação da cartilagem pelos condrócitos, como o número de receptores para interleucina-1 (IL-1) e fator de necrose tumoral (TNF). Por outro lado, os receptores do fator de crescimento (TGF) $\beta$-RII são reduzidos (ATTUR et al., 2002; BOUMEDIENE et al., 1998; WANG et al., 2003).

O desequilíbrio catabólico gera a degradação dos proteoglicanos e de colágeno tipo II e a IL-1 foi considerada uma das principais reguladoras e estimuladoras da síntese de metaloproteinases pelos condrócitos, necessitando evidentemente de outros fatores para expressar a sua atividade catabólica (MILNER; ELLIOT; CAWSTON, 2001).

No líquido sinovial da maioria dos pacientes com OA observa-se maior número de células mononucleares, como macrófagos e linfócitos T, com o concomitante aumento dos níveis de imunoglobulinas, moléculas do sistema complemento e citocinas inflamatórias (KRENN et al., 1999; NAKAMURA et al., 1999).

A membrana sinovial revela alterações características da inflamação crônica, incluindo hiperplasia de células de revestimento sinovial com infiltrado de células inflamatórias em tecidos de suporte, além de fibrose (GOLDENBERG; EGAN; COHEN, 1982). A inflamação sinovial é secundária à liberação de produtos de degradação da cartilagem para a cavidade articular (NALBANT et al., 2003).

Apesar da OA ser caracterizada por esclerose óssea subcondral, é incerto se as alterações ósseas são a causa ou a consequência das lesões, pois as propriedades da cartilagem dependem do seu leito ósseo, que pode ser afetado pela perda da função mecânica da cartilagem. Alguns estudos em animais sustentam a hipótese de que a rigidez do osso subcondral pode preceder lesões cartilaginosas (RADIN; ROSE, 1986; CARLSON et al., 1994, 1996). Evidências a favor e contra essa hipótese surgiram de estudos em modelo animal e ensaios clínicos (DEDRICK et al., 1993; BOBINAC et al., 2003), mas uma conclusão permanece indefinida.

\section{Sulfato de condroitina e glucosamina}

Os condroprotetores mais comumente utilizados são na verdade classificados como agentes condromoduladores, dos quais a função é retardar ou diminuir a progressão degenerativa. Esses agentes são considerados medicamentos de ação lenta, propondo-se três efeitos primários como condroprotetor: sustentar o aumento do metabolismo dos condrócitos e células sinoviais, inibir as enzimas degenerativas do líquido sinovial e da matriz cartilaginosa, e inibir a formação de microtrombos nos vasos da circulação que nutrem a articulação (BOOTHE, 1997).

Entre esses moduladores os seus principais componentes são o sulfato de condroitina e glucosamina. Nas formulações comerciais temos esses compostos isolados ou combinados entre si e também acrescidos de outros condroprotetores que se propõem a ter efeitos benéficos na articulação, como extratos naturais de glicosaminoglicanos derivados de moluscos, assim como algumas vitaminas (C, E), ácidos graxos (ômega 3) e minerais (Zn, $\mathrm{Cu}, \mathrm{Mn}$ ), cuja ação antioxidante pode beneficiar na redução da formação de radicais livres da articulação afetada.

A glucosamina é um aminoácido açucarado precursor de dissacarídeos dos glicosaminoglicanos (GAGs). O produto final de sua biotransformação é a uridina difosfato-N-acetilglucosamina, que é utilizada para fazer (GAGs), proteoglicanos e glicolipídeos (ROSEMAN, 2001). Em virtude de os GAGs serem os maiores componentes da cartilagem articular, a suplementação com glucosamina pode ajudar na reconstrução da cartilagem, havendo dados in vitro que suportam esta teoria (DODGE; JIMENEZ, 2003).

A glucosamina é comercialmente disponível sob três formas: hidrocloridato de glucosamina, sulfato de glucosamina e N-acetil-D-glucosamina. Elas são pequenas moléculas hidrossolúveis com pKa, que favorecem sua absorção intestinal e o transporte intracelular (KELLY, 1998).

Rev. Acad., Ciênc. Agrár. Ambient., Curitiba, v. 8, n. 3, p. 281-289, jul./set. 2010 
Os aspectos quantitativos de sua absorção são discutíveis; no entanto, estima-se que $90 \%$ da glucosamina, após a administração oral, é incorporada às proteínas do plasma e biotransformada no fígado (SETNIKAR; GIACCHETTI; ZANOLO, 1986), sendo distribuída rapidamente pelos tecidos, com tropismo pela cartilagem articular, excedendo neste local a sua concentração em relação ao plasma. Foi constatada pelos níveis de radioatividade usados para o estudo da farmacocinética. Após seis dias de uma única dose percebe-se o aumento deste índice, e com múltiplas doses há também o acúmulo da glucosamina na cartilagem (SETNIKAR; PALUMBO; CANALI, 1993).

Além do efeito benéfico no aumento da disponibilidade dos precursores dos componentes da cartilagem, a glucosamina tem outros efeitos comprovados in vitro sobre a influência da expressão de mediadores inflamatórios, reduzindo a degradação de proteoglicanos, inibindo a síntese e atividade de mediadores como as metaloproteinases, óxido nítrico e prostaglandinas. Contudo, seu efeito anabólico é limitado, não agindo sobre o colágeno tipo II, um dos principais componentes da matriz extracelular, responsável pela força de tensão da cartilagem articular (BASSLER; ROVATI; FRANCHIMONT, 1998).

O sulfato de condroitina é um glicosaminoglicano constituinte da cartilagem e, quando administrado, parece ser capaz de influenciar o metabolismo (BROWN et al., 1998). Entretanto, há controvérsias sobre a administração oral deste composto, por causa de seu tamanho de 6 a 50 kd (SAKAI; ONOSE; NAKAMURA, 2002).

O que se sabe in vitro é que, além de aumentar a síntese de glicosaminoglicanos e reduzir a degradação da glucosamina, a condroitina age inibindo a interleucina-1, com grande atividade na degradação do colágeno tipo II (BASSLER; ROVATI; FRANCHIMONT, 1998). Seus efeitos sobre a PGE2 e o óxido nítrico são mais variáveis; no entanto, têm a capacidade de aumentar a viscosidade do líquido sinovial através do aumento na concentração de ácido ácido hialurônico (RONCA et al., 1998).

\section{Estudos prospectivos}

Dobenecker, Beetz e Kienze (2002) compararam o tratamento entre dois condroprotetores, o extrato de mexilhão (perna canaliculus), rico em glicosaminoglicanos (18 cães), e o sulfato de condroitina (21 cães), e um grupo controle com placebo (19 cães), durante 84 dias. Foi um estudo randomizado duplo cego. Avaliaram os tratamentos por meio de uma escala de sinais clínicos que iam de 1 a 7 , de acordo com a severidade dos sintomas sem qualquer medicação concomitante. Não constataram diferença significativa $(\mathrm{p}<0,05)$ entre os grupos, inclusive do grupo controle com placebo. Concluíram que a possível melhora poderia ter ocorrido naturalmente, sem a ação de qualquer medicamento, uma vez que obtiveram a mesma resposta no grupo controle com o placebo.

Moreau et al. (2003) realizaram um estudo prospectivo duplo cego comparando o tratamento entre meloxicam, carprofeno, placebo e condroprotetor à base de sulfato de condroitina e glucosamina (Cosequin ${ }^{\circledR}$ ), em 71 cães ao todo, por 60 dias. A avaliação foi feita pela análise cinética, por meio de placa de força, mostrando melhora dos animais submetidos ao tratamento com AINEs, sendo que o meloxicam foi considerado o melhor, com retorno à condição normal do uso do membro afetado. Já o tratamento com condroprotetor não proporcionou melhora significativa, indicando que seu uso pode ser ineficaz para o tratamento de AAD.

Foram realizadas outras avaliações subjetivas por meio de escalas para o proprietário e ortopedista, além de um escore também realizado para o exame radiográfico de cada articulação acometida. Essa avaliação subjetiva percebeu mudanças significativas nos grupos tratados com AINEs. As avaliações de sangue fecal e bioquímico desses animais não se alteraram durante o tratamento, com exceção de um animal medicado com AINE, que manifestou intoxicação hepática. Não houve melhora com condroprotetor e questiona-se a dose recomendada pelo fabricante como uma possível causa dos resultados insatisfatórios, tanto para o proprietário quanto para o veterinário (MOREAU et al., 2003).

McCarthy et al. (2007) compararam dois tratamentos utilizando a glucosamina/sulfato de condroitina (em 16 cães) e carprofeno (19 cães) como grupo controle positivo. Esses animais tinham OA nas articulações coxofemorais ou nas escapulo-umerais. As avaliações foram feitas por um sistema de escore de sinais clínicos, reportado em outros estudos (HOLTSINGER et al., 1992; VASSEUR et al., 1995), terminando em

Rev. Acad., Ciênc. Agrár. Ambient., Curitiba, v. 8, n. 3, p. 281-289, jul./set. 2010 
70 dias de tratamento, com três avaliações durante esse período. Constataram que houve melhora nos dois tratamentos, com relação à dor e ao apoio do membro $(p<0,001)$. A resposta ao condroprotetor foi menor que ao carprofeno.

Aragon, Hofmeister e Budsberg (2007) avaliaram 16 cães com AAD, que receberam como tratamento meloxicam, carprofeno, polisulfatado glicosaminoglicanas e sulfato de condroitina. Os animais que receberam meloxicam apresentaram alto nível de conforto e moderado com o uso dos demais medicamentos.

Já em um modelo experimental de sinovite induzida, Canapp, McLaughlin e Hoskinson (1999) comprovaram uma diminuição da inflamação por meio de cintilografia nos animais tratados com condroprotetor em relação ao grupo controle com placebo. Realizaram conjuntamente exames clínicos, nos quais houve melhora da claudicação nos animais tratados.

Jonhson et al. (2001) realizaram um estudo para avaliar os efeitos de uma mistura de administração oral de sulfato de condroitina, cloridrato de glucosamina e manganês no metabolismo da cartilagem articular em cães com ligamento cruzado cranial deficiente após a transecção experimental e sua reconstrução ou não, formando quatro grupos. Foram mensuradas as concentrações de epítopos 3B3, 7D4 e glicosaminoglicanos sulfatados totais (GAG) do líquido sinovial. Encontraram um aumento considerável dos epítopos e dos glicosaminoglicanos até os cinco meses de uso do medicamento, em relação ao grupo controle sem medicação. Comprovou-se a ação moduladora desses agentes in vivo pelo aumento dos glicosaminoglicanos da matriz.

Testando a segurança dos condroprotetores, Sauvé et al. (2003) verificaram a ação sobre a função da tireoide. Realizado um estudo em 46 cães saudáveis, foram mensurados após 60 dias de tratamento os níveis de tiroxina total e livre, assim como as concentrações endógenas de tireotrofina. Verificaram que não houve diferença entre os níveis dessas concentrações, demonstrando que os condroprotetores não afetam a função tireoideana.

Com relação a alterações hematológicas, hemostáticas e bioquímicas, McNamara, Barr e Erb (1996) constataram que há uma diminuição no hematócrito, hemoglobina, neutrófilos e linfócitos. Houve redução na resposta da adenosina difosfato (ADP) e da adenosina trifosfato em relação ao colágeno. Apesar das alterações hematológicas, nenhuma alteração bioquímica ou de coagulação foi observada, e as alterações hematológicas presentes não foram de importância clínica.

\section{Constatação de evidências científicas}

No estudo de Dobenecker, Beetz e Kienze (2002), os autores compararam o tratamento entre extrato de mexilhão e sulfato de condroitina com um grupo controle com placebo. Apesar de ser um estudo randomizado duplo cego, a análise foi feita por meio de uma escala de sinais clínicos e realizada por diferentes profissionais. Outro aspecto importante a ser considerado sobre a sua conclusão é a leve melhora dos animais. Segundo a pesquisa, diferentes articulações foram incluídas e não especificaram a distribuição nessas articulações acometidas de cada grupo, sendo que nos gráficos expostos a severidade dos sintomas sofre uma distribuição heterogênea - portanto, é discutível a conclusão desse trabalho.

Moreau et al. (2003) reportam que os grupos eram similares, independentemente do tratamento, porém abordam várias articulações. Vale ressaltar que, apesar de não constatar uma melhora sobre os dados quantitativos, seu estudo foi comparativo entre AINEs, e casos severos não foram excluídos do trabalho. Dificilmente em um caso severo de osteoartrose haverá uma melhora do animal, já que o proposto do condroprotetor não é curar a artrose, mas impedir ou retardar a sua progressão por meio dos mecanismos já descritos (BUDSBERG; BARTGES, 2006). Os casos em que se acredita que os condroprotetores sejam eficazes são de leves a no máximo moderados (BEALE, 2004).

Como resultado positivo de McCarthy et al. (2007), deve ser ressaltado que houve melhora da dor e apoio. Não houve diferença na claudicação e na mobilidade articular. Tal fato é relevante, pois a falta de um grupo controle negativo com placebo poderia ter esclarecido se esta melhora foi de fato pelo condroprotetor ou não.

A comprovação do aumento dos epítopos 3B3, 7D4 e glicosaminoglicanos sulfatados totais (GAG) do líquido sinovial por Jonhson et al. (2001) deixa claro que realmente há uma resposta benéfica na modulação

Rev. Acad., Ciênc. Agrár. Ambient., Curitiba, v. 8, n. 3, p. 281-289, jul./set. 2010 
do metabolismo articular. Apesar de ser um modelo experimental e a medicação ter sido administrada logo no momento seguinte à injúria, é valido que realmente ocorreu uma resposta à medicação.

Os modelos experimentais, assim como o realizado por Canapp, McLaughlin e Hoskinson (1999), com resultados positivos demonstram fortes indícios dos benefícios do condroprotetor; contudo, por serem modelos experimentais induzidos, outros fatores podem influenciar esses resultados.

Com relação à segurança dos condroprotetores, Sauvé et al. (2003) excluíram sua interferência sobre a função tireoideana, provando que as ações de mudanças, do metabolismo articular, pretendidas por esses medicamentos, não afetam o metabolismo geral do animal - fato que assegura o uso destes produtos mesmo em casos de disfunção da glândula tireóidea.

Sabe-se que a glucosamina, sendo um açúcar, possui de fato efeito hiperglicemiante e age como inibidor de insulina (KAJINUMA et al., 1975). Tal fato foi comprovado e requer atenção, evitando essas medicações em pacientes diabéticos. Apesar das doses utilizadas nos condroprotetores serem relativamente baixas, os benefícios do condroprotetor se contrapõem ao seu possível efeito deletério nestes animais. Existem trabalhos na espécie humana que comprovam essa questão (PHAM et al., 2007).

Outro fator que chama a atenção é relativo às alterações hematológicas: os autores atentam para a questão do uso dos condroprotetores em longo prazo (McNAMARA; BARR; ERB, 1996), opondo-se aos dados obtidos por Moreau et al. (2003), os quais não constataram tais alterações sanguíneas utilizando o produto à base de sulfato de condroitina e glucosamina, por 60 dias.

Os condroprotetores possuem ação benéfica na articulação acometida pela OA, no entanto, há dados conflitantes sobre a eficácia clínica dos animais sob tratamento. Os medicamentos oferecem bom nível de segurança aos pacientes com relação aos efeitos adversos; entretanto, mais estudos são necessários para melhor esclarecimento sobre o seu uso.

\section{Referências}

AIGNER, T. et al. Anabolic and catabolic gene expression pattern analysis in normal versus osteoarthritic cartilage using complementary DNA-array technology. Arthritis and Rheumatism, v. 44, n. 12, p. 2777-2789, 2001.

ARAGON, C. L.; HOFMEISTER, E. H.; BUDSBERG, S. C. Systematic review of clinical trials of treatments for osteoarthritis in dogs. Journal of the American Veterinary Medical Association, v. 230, n. 4, p. 514-521, 2007.

ATTUR, M. G. et al. Osteoarthritis or osteoarthrosis: the definition of inflammation becomes a semantic issue in the genomic era of molecular medicine. Osteoarthritis Cartilage, v. 10, n. 1, p. 1-4, 2002.

BASSLER, C.; ROVATI, L.; FRANCHIMONT, P. Stimulation of proteoglycan production by glucosamine sulfate in chondrocytes isolated from human osteoarthritis articular cartilage in vitro. Osteoarthritis Cartilage, v. 6, n. 6, p. 427-434, 1998.

BEALE, S. B. Use of nutraceuticals and chondroprotectants in osteoarthritic dogs and cats. Veterinary Clinics Small Animal, n. 34, p. 271-289, 2004.

BLUTEAU, G. et al. Matrix metalloproteinase-1, -3, -13 and aggrecanase- 1 and -2 are differentially expressed in experimental osteoarthritis. Biochimica Biophysica Acta, v. 1526, n. 2, p. 147-158, 2001.

BOBINAC, D. et al. Changes in articular cartilage and subchondral bone histomorphometry in osteoarthritic knee joints in humans. Bone, v. 32, n. 3, p. 284-290, 2003.

BOOTHE, D. M. Nutrical ceuticals in veterinary medicine. Compendium Continuum Education Practice Veterinary, v. 19, n. 11, p. 1248-1255, 1997.

BOUMEDIENE, K. et al. Decrease of cartilage transforming growth factor-beta receptor II expression in the rabbit experimental osteoarthritis - potential role in cartilage breakdown. Osteoarthritis and Cartilage, v. 6, n. 2, p. 146149, 1998.

Rev. Acad., Ciênc. Agrár. Ambient., Curitiba, v. 8, n. 3, p. 281-289, jul./set. 2010 
BRAUNSTEIN, E. M.; BRANDT, K. D.; ALBRECHT, M. MRI demonstration of hypertrophic articular cartilage repair in osteoarthritis. Skeletal Radiology, v. 19, n. 5, p. 335-339, 1990.

BROWN, M. P. et al. Changes in sulfation patterns of chondroitin sulfate in equine articular cartilage and synovial fluid in response to ageing and osteoarthritis. American Journal Veterinary Research, v. 59, n. 6, p. 786-791, 1998.

BUDSBERG, S. C.; BARTGES, J. W. Nutrition and osteoarthritis in dogs: does it help? Veterinary Clinics of North America: Small Animal Practice, v. 36, n. 6, p. 1307-1323, 2006.

CARLSON, C. S. et al. Osteoarthritis in cynomolgus macaques: a primate model of naturally occurring disease. Journal of Orthopaedic Research, v. 12, p. 331-339, 1994.

CARLSON, C. S. et al. Osteoarthritis in cynomolgus macaques. III: effects of age, gender, and subchondral bone thickness on the severity of disease. Journal of Bone and Mineral Research, v. 11, n. 9, p. 1209-1217, 1996.

CANAPP, S. O. J. R.; MCLAUGHLIN Jr., R. M.; HOSKINSON, J. J. Scintigraphic evaluation of dogs with acute synovitis after treatment with glucosamine hydrochloride and chondroitin sulfate. American Journal Veterinary Research, v. 60 , n. 12 , p. $1552-1557,1999$.

DAVIS, M. A. Epidemiology of osteoarthritis. Clinical Geriatric Medicine, v. 4, n. 2, p. 241-255, 1988.

DEAN, D. D.; AZZO, W.; MARTEL-PELLETIER, J. Levels of metaloproteases and tissue inhibitor of metaloproteases in human ostearthritic cartilage. Journal of Rheumatology, v. 14, n. 1, p. 43-44, 1987.

DEDRICK, D. K. et al. A longitudinal study of subchondral plate and trabecular bone in cruciate-deficient dogs with osteoarthritis followed up for 54 months. Arthritis and Rheumatism, v. 36, n. 10, p. 1460-1467, 1993.

DOBENECKER, B.; BEETZ, Y.; KIENZE, E. A Placebo-controlled double-blind study on the effect of nutraceuticals (Chondroitin Sulfate and Mussel extract) in dogs with joint diseases as perceived by their owners. Journal of Nutrition, v. 132, n. 6, p. 1690S-1691S, 2002.

DODGE, G. R.; JIMENEZ, S. A. Glucosamine sulphate modulates the levels of aggrecan and matrix metalloproteinase-3 synthesized by cultured human osteoarthritis articular chondrocytes. Osteoarthritis and Cartilage, v. 11, n. 6, p. $424-432,2003$.

GINGERICH, D. A.; STROBEL, J. D. Use of client-specific outcome measures to assess treatment effects in geriatric, arthritic dogs: controlled clinical evaluation of a nutraceutical. Veterinary Therapeutics, v. 4, n. 4, p. 376-386, 2003.

GOLDENBERG, D. L.; EGAN, M. S.; COHEN, A. S. Inflammatory synovitis in degenerative joint disease. Journal of Rheumatology, v. 9, n. 2, p. 204-209, 1982.

HOLTSINGER, R. et al. The therapeutic efficacy of carprofen (Rimadyl-V) in 209 clinical cases of canine degenerative joint disease. Veterinary and Comparative Orthopedics and Traumatology, v. 5, n. 2, p. 140-144, 1992.

JOHNSTON, S. A. Osteoarthritis: joint anatomy, physiology, and pathobiology. Veterinary Clinics of North America: Small Animal Practice, v. 27, n. 4, p. 699-723, 1997.

JOHNSON, K. A. et al. Effects an orally administered mixture of chondroitin sulfate, glucosamina hydrochloryde and maganese ascorbate on synovial fluid chondroitin sulfate 3B3 and 7D4 epitopes in a canine cruciate ligament transection model of osteoarthritis. Osteoarthritis and Cartilage, v. 9, n. 1, p. 14-21, 2001.

KAJINUMA, H. et al. Effects of glucosamine on insulin and glucagon secretion in dogs and ducks. Endocrinology Japanese, v. 22, n. v. 6, p. 517-23, 1975.

KELLY, G. S. The role of glucosamine sulfate and chondroitin sulfates in the treatment of degenerative joint disease. Alternative Medicine Review, v. 3, n. 1, p. 27-39, 1998.

KUTT'NER K.; GOLDBERG V. M. Osteoarthritic disorders. Rosemont: American Academy of Orthopaedic Surgeons, 1995.

Rev. Acad., Ciênc. Agrár. Ambient., Curitiba, v. 8, n. 3, p. 281-289, jul./set. 2010 
KIRSCH, T.; SWOBODA, B.; NAH, H. Activation of annexin II and V expression, terminal differentiation, mineralisation and apoptosis in human osteoarthritic cartilage. Osteoarthritis and Cartilage, v. 8, n. 4, p. 294-302, 2000.

KRENN, V. et al. Molecular $\operatorname{IgV}(\mathrm{H})$ analysis demonstrates highly somatic mutated B cells in synovitis of osteoarthritis: a degenerative disease is associated with a specific, not locally generated immune response. Laboratory Investigation, v. 79 , n. 11, p. 1377-1384, 1999.

KUSUZAKI, K. et al. DNA cytofluorometric analysis of chondrocytes in human articular cartilages under normal aging or arthritic conditions. Osteoarthritis and Cartilage, v. 9, n. 7, p. 664-670, 2001.

LIPOWITZ, J. A. Afecção articular degenerativa. In: SLATTER, D. Manual de cirurgia de pequenos animais. 2. ed. São Paulo: Manole, 1998. v. 2, p. 2266-2273.

LIPPIELLO, L. et al. In vivo chondroprotection and metabolic synergy of glucosamine and chondroitin sulfate. Clinical Orthopaedics and Related Research, v. 381, p. 229-240, 2000.

McCARTHY, G. et al. Randomised double-blind, positive-controlled trial to assess the efficacy of glucosamine/chondroitin sulfate for the treatment of dogs with osteoarthritis. Veterinary Journal, v. 174, n. 1, p. 54-61, 2007.

McNAMARA, P.; BARR, S.; ERB, H. Hematologic, hemostatic, and biochemical effects in dogs receiving an oral chondroprotective agent for thirty days. American Journal of Veterinary Research, v. 57, n. 9, p. 1390-1394, 1996.

MILNER, J. M.; ELLIOTT, S. F.; CAWSTON, T. E. Activation of procollagenases is a key control point in cartilage collagen degradation: interaction of serine and metalloproteinase pathways. Arthritis and Rheumatism, v. 44, n. 9 , p. 2084-2096, 2001.

MOREAU, M. et al. Clinical evaluation of a nutraceutical, carprofen and meloxicam for treatment of dogs with osteoarthritis. Veterinary Records, v. 152, n. 11, p. 323-329, 2003.

NALBANT, S. et al. Synovial fluid features and their relations to osteoarthritis severity: new findings from sequential studies. Osteoarthritis and Cartilage, v. 11, n. 1, p. 50-54, 2003.

NAKAMURA, H. et al. T-cell mediated inflammatory pathway in osteoarthritis. Osteoarthritis and Cartilage, v. 7, n. 4, p. 401-402, 1999.

NEIL, K. M.; CARON, J. P.; ORTH, M. W. The role of glucosanibe and chondroitin sulfate in treatment for and prevention of osteoarthritis in animals. Journal of the American Veterinary Medical Association, v. 226, n. 7, p. 1079-1087, 2005.

PHAM T. C. A. et al. Oral glucosamine in doses used to treat osteoarthritis worsens insulin resistance. American Journal of the Medical Science, v. 333, n. 6, p. 333-339, 2007.

RADIN, E. L.; ROSE, R. M. Role of subchondral bone in the initiation and progression of cartilage damage. Clinica Orthopaedica, v. 213, p. 34-40, 1986.

RONCA, F. et al. Anti-inflamatory activity of chondroitin sulfate. Osteoarthritis and Cartilage, v. 6, p. 14-21, 1998. Suppl A.

ROSEMAN, S. Reflections on glycobiology. Journal of Chemical Biology, v. 276, n. 45, p. 1527-1542, 2001.

SAKAI, S.; ONOSE, J.; NAKAMURA, H. Pretreatment procedure for the microdertermination of chondroitin sulfate in plasma and urine. Analytical Biochemistry, v. 302, n. 2, p. 169-174, 2002.

SAUVÉ, F. et al. Effects of oral administration of meloxicam, carprofen, and a nutraceutical on thyroid function in dogs with osteoarthritis. Canine Veterinary Journal, v. 44, n. 6, p. 474-479, 2003.

SETNIKAR, L.; GIACCHETTI, C.; ZANOLO, G. Pharmacokinetics of glucosamine in dog and man. ArzeneimForsch Drug Research, v. 36, n. 4, p. 729-35, 1986.

Rev. Acad., Ciênc. Agrár. Ambient., Curitiba, v. 8, n. 3, p. 281-289, jul./set. 2010 
SETNIKAR, L.; PALUMBO, R.; CANALI, S. Pharmacokinetics of glucosamine in man. Arzeneimittleforschung, v. 43, n. 10, p. 1109-1113, 1993.

TETLOW, L. C.; ADLAM, D. J.; WOOLLEY, D. E. Matrix metalloproteinase and proinflammatory cytokine production by chondrocytes of human osteoarthritic cartilage: associations with degenerative changes. Arthritis and Rheumatism, v. 44, n. 3, p. 585-594, 2001.

VASSEUR, P. et al. Randomized, controlled trial of the efficacy of carprofen, a nonsteroidal anti-inflammatory drug, in the treatment of osteoarthritis in dogs. Journal of the American Veterinary Medical Association, v. 206, n. 6, p. 807-811, 1995.

WANG, J. et al. Homeostasis of the extracellular matrix of normal and osteoarthritic human articular cartilage chondrocytes in vitro. Osteoarthritis and Cartilage, v. 11, n. 11, p. 801-809, 2003.

YASUDA, T.; POOLE, A. R. A fibronectin fragment induces type II collagen degradation by collagenase through an interleukin-1-mediated pathway. Arthritis and Rheumatism, v. 46, n. 1, p. 138-148, 2002.

Recebido: 10/11/2009

Received: $11 / 10 / 2009$

Aprovado: 03/05/2010

Approved: 05/03/2010

Rev. Acad., Ciênc. Agrár. Ambient., Curitiba, v. 8, n. 3, p. 281-289, jul./set. 2010 